\title{
Stability of Stochastic Systems with Jumps
}

\author{
E.K. BOUKAS* and H. YANG
}

Mechanical Engineering Department, École Polytechnique de Montréal, P.O. Box 6079, Station "centre-ville", Montréal, Québec, Canada, H3C $3 A 7$

(Received 15 January 1996; Revised 30 April 1996)

This paper deals with stochastic stability of systems with Markovian jumps and Brownian motion. Mainly, we present sufficient conditions for quadratic stabilization of Ito type stochastic linear and nonlinear systems with Markovian jumps and Brownian motion using state feedback control. We also prove the guaranteed cost property of the proposed control strategy for the linear case.

Keywords: Systems with Markovian jumps and Brownian motion; Stochastic Stability; Markov Process; Ito Differential Equation; Lyapunov Function.

Classification Categories: 43E15

\section{INTRODUCTION}

Due to the need for modeling real world dynamic systems which may experience abrupt changes in their structure and parameters caused by component failures or repairs, changing environmental disturbances and subsystem interconnections, linear systems with Markovian jumping parameters have received recently a remarkable attention (see for example $\mathrm{Ji}$ and Chizeck [1], Wonham [2], Sworder [3], Mariton and Bertrand [4], Mariton [5, 6], Boukas [7] and references therein). For systems with

*Corresponding author. E-mail address: Boukas@ anas.meca.polymtl.ca 
Brownian motion, there exists in the literature many references dealing with the stochastic stability, see for example the works of Florchinger [8], Kushner [9], Willems and Willems [10], Khasminskii [11] and the references therein.

Most of the presently used analysis and design tools for feedback control systems presuppose that a sufficiently linear model of the process to be controlled is available. This model is not the true representation of the physical system under study since we always make a trade off between the model accuracy and the model complexity. The next step should be the use of a robust control law which enables the designer to explicitly consider the discrepancies between the model and the real process. Theoretically, this approach allows the designer to get results that are not only valid for the approximate model but do hold with certainty for the real process also.

In the control literature, the uncertain systems has received much attention. The quantity of papers and books dealing with this type of problem justifies from the practical point of view the interest of this topics. The Brownian motion, known also as Wiener process is the most studied and the most fascinating process. Many results for linear systems with Brownian motion exist. But to the best of our knowledge no results for linear systems with jumps and Brownian motion exists. Our primary aim is then to study the stochastic stability of this class of systems.

The main idea of this paper consists to use the Lyapunov function techniques to establish a sufficient condition of stability for systems with Markovian jumps and Brownian motion. In linear case, we prove that if the diffusion coefficient is upper bounded by a positive definite matrix (i.e. for two matrices $C$ and $D$, when we say that the matrix inequality $C<D$ holds, this means that $C-D$ is a nonnegative matrix) then the system under consideration is stochastically stable. We state and prove the guaranteed cost property of the proposed control policy for linear system. We also establish a stochastic stability result for nonlinear system.

The paper is organized as follows. In section 2, we consider the linear system. Three theorems are presented and proved. Theorem 2.1 gives sufficient condition of stability of the system under consideration. Theorem 2.2 is an application of Theorem 2.1. Theorem 2.3 establishes the guaranteed cost property. In section 3 , we consider the nonlinear system. A stochastic stability result is given. The contribution of the paper is summarized in section 4 . 


\section{LINEAR SYSTEM}

In this section, we will give a description of the class of systems under study. We will also state and prove the stochastic stability results.

\subsection{Problem Statement}

Let us consider a class of linear systems with Markovian jumps and Brownian motion described by:

$$
\begin{gathered}
d x(t)=[A(\xi(t)) x(t)+B(\xi(t)) u(t)] d t+\sum_{i=1}^{k} g_{i}(\xi(t)) x(t) d W_{i} \\
x(0)=x_{0}
\end{gathered}
$$

where the vector $x(t) \in I R^{n}$ is the state of the system and the vector $u(t) \in$ $I R^{m}$ is the control, and the vector $W_{i}(t),(i=1, \ldots, k)$ denotes the disturbance, assumed to be standard Brownian motion. The term $\sum_{i=1}^{k} g_{i}(\xi(t)) x$ $(t) d W_{i}$ represents the system uncertainty. The parameter $\xi(t)$ represents a continuous discrete-state Markov process taking values in a finite set $\mathrm{B}=$ $\{1,2, \ldots, s\}$ with transition probability, $\operatorname{Pr}\{\xi(t+\delta t)=\beta \mid \xi(t)=\alpha\}$, given by:

$$
\operatorname{Pr}\{\xi(t+\delta t)=\beta \mid \xi(t)=\alpha\}=\left\{\begin{array}{l}
q_{\alpha \beta} \delta t+o(\delta t), \quad \text { if } \alpha \neq \beta \\
1+q_{\alpha \alpha} \delta t+o(\delta t), \quad \text { if } \alpha=\beta
\end{array}\right.
$$

In this relation, $q_{\alpha \beta}$ stands for the transition probability rate from mode $\alpha$ to mode $\beta$ and satisfies the following relations:

$$
\begin{gathered}
q_{\alpha \beta} \geq 0 \\
q_{\alpha \alpha}=-\sum_{\beta \in \mathrm{B}, \alpha \neq \beta} q_{\alpha \beta}
\end{gathered}
$$

In Eq. (1), $A(\xi(t)), B(\xi(\mathrm{t}))$ and $g_{i}(\xi(t))$ are appropriately dimensioned matrices. These matrices are constant for a given value of $\xi(t)$. 
In this paper, we will assume that the Markov process $\xi(t)$ and the noise processes $W_{i}(t)$ are independent. We also assume that perfect state information is available for feedback.

In order to control the system described by Eq. (1), we use the optimal control approach. The optimization problem consists of minimizing the cost function, $J$ defined by:

$$
J=\mathbf{E}\left\{\int_{0}^{\infty} x^{\prime}(t) Q(\xi(t)) x(t)+u^{\prime}(t) R(\xi(t)) u(t) d t\right\}
$$

subject to the constraints (1)-(2).

In relation (6), the cost weighting matrices $R(\xi(t))$ and $Q(\xi(t))$ are symmetric with $R(\xi(t))$ positive definite and $Q(\xi(t))$ positive semi-definite for each $\xi(t)$.

In the following, we will refer to the jump linear systems described by the following differential equation:

$$
\begin{gathered}
d x(t)=[A(\xi(t)) x(t)+B(\xi(t)) u(t)] d t \\
x(0)=x_{0}
\end{gathered}
$$

as a nominal system of system (1).

Let $x\left(t, x_{0}, \alpha, u\right)$ represent the corresponding solution of system (1) at time $t$ when the control $u($.$) is used and the initial conditions are respec-$ tively $x_{0}$ and $\alpha$.

The stability is always the first requirement for any control design. For our class of systems, we will use the concept of mean square stochastic stability given by the following definition:

Definition 2.1 The system (1)-(3) is said to be stochastically stabilizable if, for all finite $x_{0} \in I R^{n}$ and $\alpha \in B$, there exists a state feedback control with finite constant matrix $K(\alpha)$, i.e. $u(t)=-K(\alpha) x(\mathrm{t})$, such that the following relation holds:

$$
\lim _{\mathrm{T} \rightarrow \infty} E_{u(.)}\left\{\int_{0}^{T} x^{\prime}\left(t, x_{0}, e \alpha, u\right) x\left(t, x_{0}, \alpha, u\right) d t \mid x_{0}, \alpha\right\} \leq x_{0}^{\prime} \tilde{P} x_{o}
$$

with $\tilde{P}$ is a symmetric and positive-definite matrix 
In the remainder of this paper, we assume that the nominal system described by Eq. (1) is stochastically stable and the first component of the state vector, i.e. $x(t)$ in each mode is assumed to be available for feedback.

It was established (see Ji and Chizeck [1] (1990)) that the solution of the optimization problem without the disturbance, $W(t)$, is given by

$$
u(\alpha, t)=-L_{0}(\alpha) x(t) \text {, when } \xi(t)=\alpha
$$

where

$$
L_{0}(\alpha)=R^{-1}(\alpha) B^{\prime}(\alpha) K_{0}(\alpha)
$$

The matrix $K_{0}(\alpha)$ is the solution of the following coupled algebraic Riccati equations:

$$
\begin{aligned}
A^{\prime}(\alpha) K_{0}(\alpha)+ & K_{0}(\alpha) A(\alpha)-K_{0}(\alpha) B(\alpha) R^{-1}(\alpha) B^{\prime}(\alpha) K_{0}(\alpha)+q_{\alpha \alpha} K_{0}(\alpha) \\
& +\sum_{\beta=1, \beta \neq \alpha}^{s} q_{\alpha \beta} K_{0}(\beta)+Q(\alpha)=0, \forall \alpha \in \mathrm{B}
\end{aligned}
$$

In the rest of this section, we will construct the control law that stabilizes the system when the disturbance is upper bounded and also discuss the guaranteed cost property.

\subsection{Main Results}

In this subsection, we first give a theorem which states the condition for the mean square stability of the system described by Eqs. (1)-(3).

THEOREM 2.1 If there exist positive definite matrices $Q(\alpha)$ and $R(\alpha)$ such that the following nonlinear coupled matrix equation has a positive definite solution $K(\alpha)$,

$$
\begin{gathered}
A^{\prime}(\alpha) K(\alpha)+K(\alpha) A(\alpha)-K(\alpha) B(\alpha) R^{-1}(\alpha) B^{\prime}(\alpha) K(\alpha)+q_{\alpha \alpha} K(\alpha) \\
\quad+\sum_{i=1}^{k} g_{i}^{\prime}(\alpha) K(\alpha) g_{i}(\alpha)+\sum_{\beta=1, \beta \neq \alpha}^{s} q_{\alpha \beta} K(\beta)+Q(\alpha)=0, \alpha \in \mathrm{B}
\end{gathered}
$$


Then the system (1)-(3) is stochastically stable under the feedback control law $u=-L(\alpha) x(t)$ with

$$
L(\alpha)=R^{-1}(\alpha) B^{\prime}(\alpha) K(\alpha)
$$

Proof Let the Lyapunov function $V(x, \alpha)$ be:

$$
V(x, \alpha)=x^{\prime}(t) K(\alpha) x(t)
$$

Consider weak infinitesimal operator $\tilde{A}$ of the joint process $(\xi(t), x(t))$ which is the natural analogue of the deterministic derivative of the Lyapunov function and is defined as follows:

$$
\begin{gathered}
\tilde{A} V(x, \alpha)=\lim _{h \rightarrow 0} \frac{1}{h}[E\{V(x(t+h), \xi(t+h)) \mid x(t), \xi(t) \\
=\alpha\}-V(x(t), \xi(t)=\alpha)]
\end{gathered}
$$

The weak infinitesimal operator is then given by:

$$
\begin{gathered}
\tilde{A} V(x, \alpha)=x^{\prime}(t)\left\{\left[A(\alpha)-B(\alpha) R^{-1}(\alpha) B^{\prime}(\alpha) K(\alpha)\right]^{\prime} K(\alpha)\right. \\
\left.+K(\alpha)\left[A(\alpha)-B(\alpha) R^{-1}(\alpha) B^{\prime}(\alpha) K(\alpha)\right]+\sum_{\beta \in B} \mathrm{q}_{\alpha \beta} \mathrm{K}(\beta)\right\} \mathrm{x}(\mathrm{t}) \\
+\mathrm{x}^{\prime}(\mathrm{t})\left[\sum_{\mathrm{i}=1}^{\mathrm{k}} \mathrm{g}_{\mathrm{i}}^{\prime}(\alpha) \mathrm{K}(\alpha) \mathrm{g}_{\mathrm{i}}(\alpha)\right] \mathrm{x}(\mathrm{t})
\end{gathered}
$$

It is easy to see that

$$
\tilde{A} V(x, \alpha)=x^{\prime}(t)\left[Q(\alpha)+L^{\prime}(\alpha) R(\alpha) L(\alpha)\right] x(t)
$$

Therefore,

$$
\frac{\tilde{A} V(x, \alpha)}{V(x, \alpha)} \leq-\frac{\lambda_{\min }\left[Q(\alpha)+K(\alpha) B(\alpha) R^{-1}(\alpha) B^{\prime}(\alpha) K(\alpha)\right]}{\lambda_{\max }(K(\alpha))}
$$

Then by Dynkin's formula and the Bellman-Gronwall lemma for all $\alpha \epsilon$ B. it follows that: 


$$
E[V(x, \alpha)] \leq \exp (-\gamma t) V\left(x_{0}, \alpha\right)
$$

where

$$
\gamma=\min _{\alpha \in \mathrm{B}}\left\{\frac{\lambda_{\min }\left[Q(\alpha)+K(\alpha) B(\alpha) R^{-1}(\alpha) B^{\prime}(\alpha) K(\alpha)\right]}{\gamma_{\max }[K(\alpha)]}\right\}
$$

Thus, after some manipulations (see Ji and Chizeck [1]) the Eq. (20) gives:

$$
\lim _{T \rightarrow \infty} E\left[\int_{0}^{T} x^{\prime}(t) K(\alpha) x(t) d t \mid x_{0}, \xi(0)=\alpha\right] \leq \frac{1}{\gamma} x_{0}^{\prime} K(\alpha) x_{0}
$$

Since $\mathrm{K}(\alpha)>0$ for each $\alpha \in B$, thus

$$
\lim _{T \rightarrow \infty} E\left[\int_{0}^{T} x^{\prime}(t) x(t) d t \mid x_{0}, \xi(0)=\alpha\right] \leq x_{0}^{\prime} \max _{\alpha \in \mathrm{B}} \frac{K(\alpha)}{\gamma\|K(\alpha)\|} x_{0}
$$

which proves the theorem.

Notice that Eq. (13) is nonlinear and its solution is not easy to obtain. Next theorem gives a sufficient condition that avoids the solution of Eq. (13).

THEOREM 2.2 Let $K_{O}(\alpha)$ denote the solution of the coupled algebraic Riccati equation (12). If the nominal system (7) is stochastically stable and there exists a positive definite matrices $Q^{*}(\alpha)$ such that the following relation holds:

$$
\sum_{i=1}^{k} g_{i}^{\prime}(\alpha) K_{0}(\alpha) g_{i}(\alpha) \leq Q^{*}(\alpha)
$$

Then the system (1) is stochastically stable.

Proof Let $K_{0}(\alpha)$ be the solution of the coupled algebraic Riccati equations (12), with $Q(\alpha)=Q^{*}(\alpha)+\epsilon Q_{1}$, where $Q_{1}$ is a positive definite symmetric matrix and $\epsilon$ is a positive number. It is easy to see that $K_{0}(\alpha)$ is solution of the following equation: 


$$
\begin{aligned}
A^{\prime}(\alpha) K_{0}(\alpha)+ & K_{0}(\alpha) A(\alpha)-K_{0}(\alpha) B(\alpha) R^{-1}(\alpha) B^{\prime}(\alpha) K_{0}(\alpha)+q_{\alpha \alpha} K_{0}(\alpha) \\
& +\sum_{i=1}^{k} g_{i}^{\prime}(\alpha) K_{0}(\alpha) g_{i}(\alpha)+\sum_{\beta=1, \beta \neq \alpha}^{s} q_{\alpha \beta} K_{0}(\beta) \\
=- & Q_{1}-\left[Q^{*}(\alpha)-\sum_{i=1}^{k} g_{i}^{\prime}(\alpha) K_{0}(\alpha) g_{i}(\alpha)\right], \quad \alpha \in \mathrm{B}
\end{aligned}
$$

Notice that $\epsilon \mathrm{Q}_{1}+\mathrm{Q}^{*}(\alpha)-\sum_{i=1}^{k} g_{i}^{\prime}(\alpha) K_{0}(\alpha) \mathrm{g}_{\mathrm{i}}(\alpha)>0$, and Eq. (25) is an equation of type (13), therefore from theorem 2.1 the result follows.

Remark The control law, $u\left(\alpha,(x(t))=-K_{0}(\alpha) x(t)\right.$, stabilizes both the nominal and the uncertain systems.

The following theorem states the condition for the guaranteed cost property.

THEOREM 2.3 Assume that the system described by the state equation (1)-(3) is governed by the control law $u=-L(\alpha) x(t)$ with $L(\alpha)$ given by (13), then the value of the performance index (6) for the system is given by the function:

$$
S\left(x_{0}, \xi(0)=\alpha\right)=x_{0}^{\prime} K(\alpha) x_{0}
$$

where $K(\alpha)$ is the matrix solution of equations (12).

Proof Using Lyapunov function (15), the value of the performance index (6) for the system (1)-(3) may be estimated as follows:

$$
\begin{aligned}
& E\left\{\int_{0}^{\infty}\left[x^{\prime}(t) Q(\xi(t)) x(t)+u^{\prime}(t) R(\xi(t)) u(t)\right] d t\right\} \\
& \quad=E\left\{\int_{0}^{\infty}\left[x^{\prime}(t)\left\{Q(\xi(t))+K(\xi(t)) B(\xi(t)) R^{-1}(\xi(t)) B^{\prime}(\xi(t)) K(\xi(t))\right\} x(t)\right] d t\right\} \\
& \quad \leq-\int_{0}^{\infty} \tilde{A} V(x(t), \xi(t)) d t \\
& \quad=E \int_{0}^{\infty}-\lim _{h \rightarrow 0} \frac{1}{h}\left\{E\left[V(x(t+h), \xi(t+h)) \mid x(t), \xi(t), W_{i}(t)\right]\right. \\
& \quad-V(x(t), \xi(t))\} d t
\end{aligned}
$$




$$
\begin{aligned}
& =E\left\{-\lim _{h \rightarrow 0} \sum_{k=0}^{\infty} h \frac{1}{h}\left\{E\left[V(x((k+1) h), \xi((k+1) h)) \mid x(k h), \xi(k h), W_{i}(k h)\right]\right.\right. \\
& -V(x(k h), \xi(k h))\}\} \\
& =-E\left\{V\left(x(t=\infty), \xi(t=\infty) \mid x_{0}, \xi(0)=\alpha\right)\right\}+V\left(x_{0}, \alpha\right)
\end{aligned}
$$

From inequality (19) the first term on the right-hand side of (27) is zero. Thus since the left-hand side of inequality (27) is the cost to go from $\left(x_{0}\right.$, $\xi(0)=\alpha)$ for the system (1)-(3), and the right-hand side equals the Lyapunov function:

$$
V\left(x_{0}, \xi(0)=\alpha\right)=x_{0}{ }^{\prime} K(\alpha) x_{0}
$$

The Theorem 2.3 is then proven.

\section{NONLINEAR SYSTEM}

In this section, we will discuss the stochastic stability of nonlinear systems. Unlike the linear system, the main difficulty here is how to construct the desired control law.

\subsection{System and Assumptions}

We consider the class of nonlinear systems described by:

$$
d x(t)=[A(x(t), \xi(t))+B(x(t), \xi(t)) u(t)] d t+\sum_{i=1}^{k} g_{i}(x(t), \xi(t)) d W_{i}
$$

where the vector $x(t) \in I R^{n}$ is the state of the system and the vector $u(t) \epsilon$ $I R^{m}$ is the control. $A(x, \xi)$ and $B(x, \xi)$ are vectors of appropriate dimensions. $g_{i}(x(t), \xi(t))$ is a $n$-dimensional vector. $W_{i}(t),(i=1, \ldots, k)$ are independent Brownian motion. $\xi(t)$ represents the continuous discrete-state Markov process taking values in a finite set $B=\{1,2, \ldots, s\}$ with transition probability, $\operatorname{Pr}\{\xi(t+\delta t)=\beta \mid \xi(t)=\alpha\}$, given by (3). 
In the rest of this subsection, we will give some assumptions which will allow us to state the sufficient condition for the the stochastic stability of the class of nonlinear systems with Markovian jumps and Brownian motion. Our first assumption is introduced to guarantee the existence of solutions of the state equations.

Assumption 3.1. For each $\alpha \in B, \mathrm{~A}(),. B($.$) , and \mathrm{g}_{\mathrm{i}}($.$) are Lipschitz con-$ tinuous in $(\mathrm{x}, \mathrm{a})$, and $\mathrm{g}_{\mathrm{i}}($.$) is a predictable process.$

Let the stochastic Lyapunov function $V(\alpha, x)$ be defined by

$$
V(\alpha, x)=x^{\prime} P(\alpha) x
$$

where $P(\alpha)$ is a symmetric positive definite matrix.

AsSUMPTION 3.2. Let the weak infinitesimal operator of the process $\{\xi(t)$, $x(t), t \in[0, T]\}$ (where $x(t)$ is the solution of the nominal model) be defined by:

$$
A_{0} V(\alpha, x)=\nabla_{x}^{\prime} V(\alpha, x) A(x, \alpha, t)+\sum_{\beta \in B} \mathrm{q}_{\alpha \beta} \mathrm{V}(\beta, \mathrm{x})
$$

where $\nabla_{x}^{\prime}$ denotes the transpose of gradient operation. We also require that there is a constant $\gamma_{1}>0$ such that

$$
A_{0} V(\alpha, x) \leq-\gamma_{1} V(\alpha, x)
$$

The next assumption is about the boundedness of the diffusion coefficients.

Assumption 3.3. Assume that the quantity $\frac{\sum_{i=1}^{k} g_{i}^{\prime}(x(t), \alpha) \mathrm{K}(\alpha) g_{i}(x(t), \alpha)}{\left\|\mathrm{B}^{\prime}(x(t), \alpha) \nabla_{x} \mathrm{~V}(\alpha, x)\right\|^{2}}$ satisfies the following:

$$
\frac{\sum_{i=1}^{k} g_{i}^{\prime}(x(t), \alpha) K(\alpha) g_{i}(x(t), \alpha)}{\left\|B^{\prime}(x(t), \alpha) \nabla_{x} V(\alpha, x)\right\|^{2}}\left\{\begin{array}{l}
\leq \gamma(x, \alpha, t), \text { if }\left\|B^{\prime}(x(t), \alpha) \nabla_{x} V(\alpha, x)\right\|^{2} \neq 0 \\
=0, \text { if }\left\|B^{\prime}(x(t), \alpha) \nabla_{x} V(\alpha, x)\right\|^{2}=0
\end{array}\right.
$$

$\gamma(x, \alpha, t)$ is a nonnegative function of $x, \alpha$ and $t$. 


\subsection{Controller Construction and the Concept of Stability}

In this section, we proceed with the construction of the control law $u^{*}($. which will later be shown to guarantee the stochastic stability of the class of systems under study.

For each $\alpha \in B$, let us choose any nonnegative function $\gamma(x, \alpha, t)$ that is continuous in $(x, t)$ and satisfying the following:

$$
\gamma(x, \alpha, t)\left\{\begin{array}{l}
\geq \frac{\sum_{i=1}^{k} g_{i}^{\prime}(x(t), \alpha) K(\alpha) g_{i}(x(t), \alpha)}{\left\|B^{\prime}(x(t), \alpha) \nabla_{x} V(\alpha, x)\right\|^{2}} \text { when }\left\|B^{\prime}(x(t), \alpha) \nabla_{x} V(\alpha, x)\right\|^{2} \neq 0 \\
=0 \text { otherwise }
\end{array}\right.
$$

We define the controller by the following expression:

$$
u^{*}(x, \alpha, t):=-\gamma(x, \alpha, t) B^{\prime}(x, \alpha, t) \nabla_{x} V(\alpha, x)
$$

\subsection{Main Result}

In this section, we will prove that the control law given by Eq. (35) stabilizes the class of nonlinear systems with Markovian jumps and Brownian motion under study.

THEOREM 3.1. With assumptions 3.1 and 3.3, and the control law (35), the stochastic nonlinear system with jumps (29) and (2) is stochastically stable.

Proof Consider the weak infinitesimal operator $\tilde{\mathrm{A}}$ of the process $\{\xi, x(t)$, $\left.W_{i}(t), t \in[0, T]\right\}$, which is given by (see Kushner [9]):

$$
\begin{aligned}
\tilde{A} V(\alpha, x)= & \nabla_{x}^{\prime} V(\alpha, x)\left\{A(x, \alpha, t)+B(x, \alpha, t) u^{*}\right\}+\sum_{\beta \in B} q_{\alpha \beta} V(\beta, x) \\
& +\sum_{i=1}^{k} g_{i}^{\prime}(x(t), \alpha) K(\alpha) g_{i}(x(t), \alpha) \\
= & A_{0} V(\alpha, x)-\nabla_{x}^{\prime} V(\alpha, x) B(x, \alpha, t) \gamma(x, \alpha, t) B^{\prime}(x, \alpha, t) \nabla_{x} V(\alpha, x)
\end{aligned}
$$




$$
\begin{aligned}
& +\sum_{i=1}^{k} g_{i}^{\prime}(x(t), \alpha) K(\alpha) g_{i}(x(t), \alpha) \\
= & A_{0} V(\alpha, x)-\gamma(x, \alpha, t)\left\|B^{\prime}(x, \alpha, t) \nabla V(\alpha, x)\right\|^{2} \\
& +\sum_{i=1}^{k} g_{i}^{\prime}(x(t), \alpha) K(\alpha) g_{i}(x(t), \alpha) \\
\leq & A_{0} V(\alpha, x) \leq-\gamma_{1} V(\alpha, x)
\end{aligned}
$$

Then by the same argument of the end of the proof of Theorem 2.1, the proof of this theorem is completed.

\section{CONCLUSION AND FUTURE DIRECTIONS}

In this paper we have proposed a stabilization procedure for the class of linear and nonlinear systems with Markovian jumps and Brownian motion. We designed a control strategy which ensures both stability and guaranteed cost property for the class of linear systems with Markovian jumps and Brownian motion, we also designed a control law which guarantees the stability of the class of nonlinear systems. The results have been obtained under the assumptions that the continuous Markov process $\xi(t)$ has a finite state space and the perfect observability of the continuous state $x(t)$ in each mode. Other problems can be investigated for instance the design of a robust controller, the case of imperfect state, the case with uncertainty on the jumps.

\section{Acknowledgements}

Research of the first author was supported by the Natural Sciences and Engineering Research Council of Canada under grants OGP0036444.

\section{References}

[1] Y. Ji, and H. Chizek. Controllability, Stability, and Continuous-Time Markovian Jump Linear Quadratic Control. IEEE Trans. Automat. Control AC-35(7) (1990), 777-788.

[2] W. M. Wonham. Random Differential Equations in Control Theory. In Probabilistic Methods in Applied Mathematics Vol. 2, A.T. Bharucha-Reid Ed., New York, 1970, 131-212. 
[3] D. Sworder. Feedback Control of a Class of Linear Systems with Jump Parameters. IEEE Trans. Automat. Control AC-14 (1969), 9-14.

[4] M. Mariton, and P. Bertrand. Robust Jump Linear Quadratic Control: A Mode Stabilizing Solution. IEEE Trans. Automat. Control AC-30 (1985), 1145-1147.

[5] M. Mariton. On Controllability of Linear Systems with Stochastic Jump Parameters. IEEE Trans. Automat. Control AC-31 (1986), 680-683.

[6] M. Mariton. Jump Linear Control Systems. New York: Marcel-Dekker, 1990.

[7] E. K. Boukas. Control of Systems with Controlled Jump Markov Disturbances. Control Theory and Advanced Technology 9(2) (1993), 577-595.

[8] P. Florchinger. On the Stabilization of Homogeneous Control Stochastic Systems. IEEE Decision and Control 1 (1993), 855-856.

[9] H. J. Kushner. Stochastic Stability and Control New York: Academic Press, 1967.

[10] J. L. Willems, and J.C. Willems. Feedback Stabilizability for Stochastic Systems with State and Control Dependent Noise. Automatica 12 (1976), 277-283.

[11] R. Z. Khasminskii. Stochastic Stability of Differential Equations. Sijthoff \& Noordhoff, Alphen aan den Rijn, 1980. 


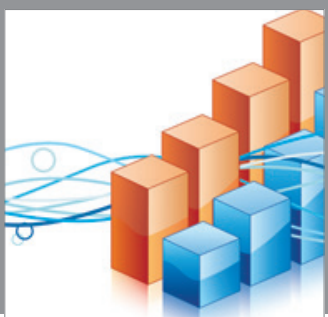

Advances in

Operations Research

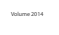

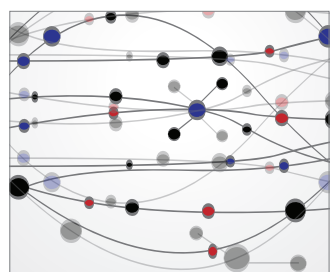

\section{The Scientific} World Journal
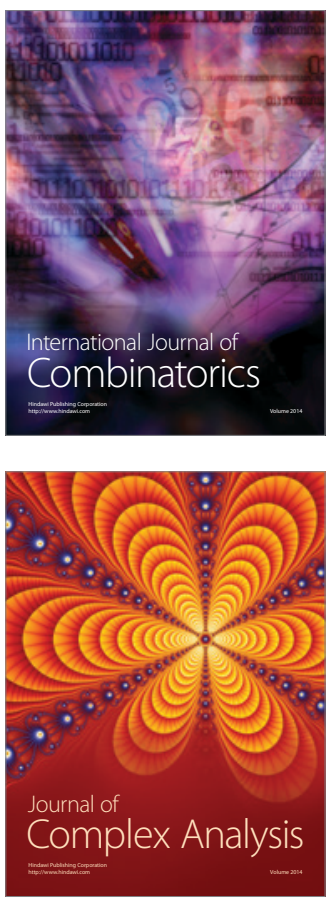

International Journal of

Mathematics and

Mathematical

Sciences
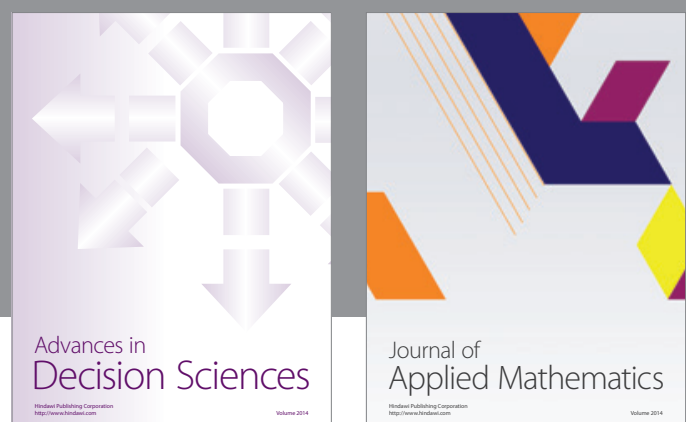

Journal of

Applied Mathematics
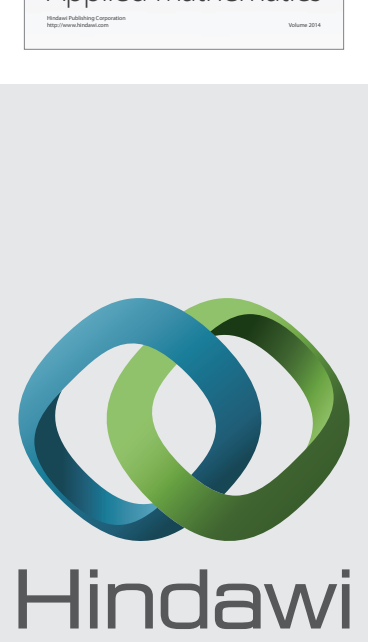

Submit your manuscripts at http://www.hindawi.com
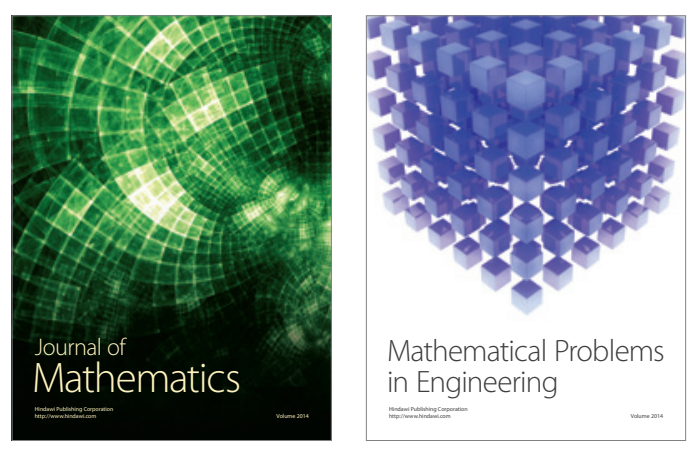

Mathematical Problems in Engineering
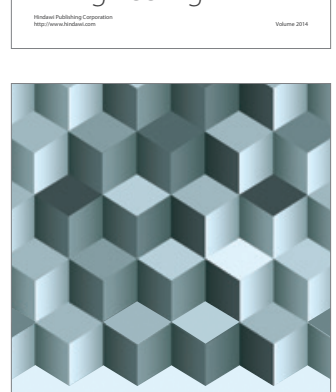

Journal of

Function Spaces
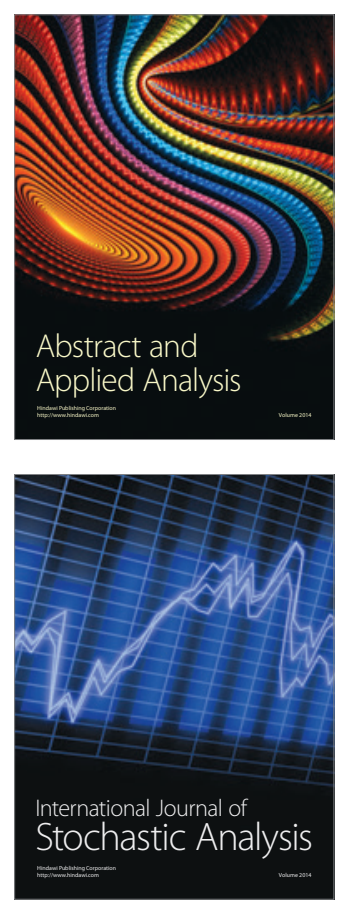

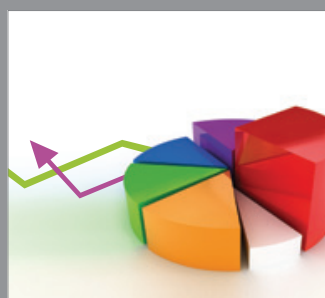

ournal of

Probability and Statistics

Promensencen
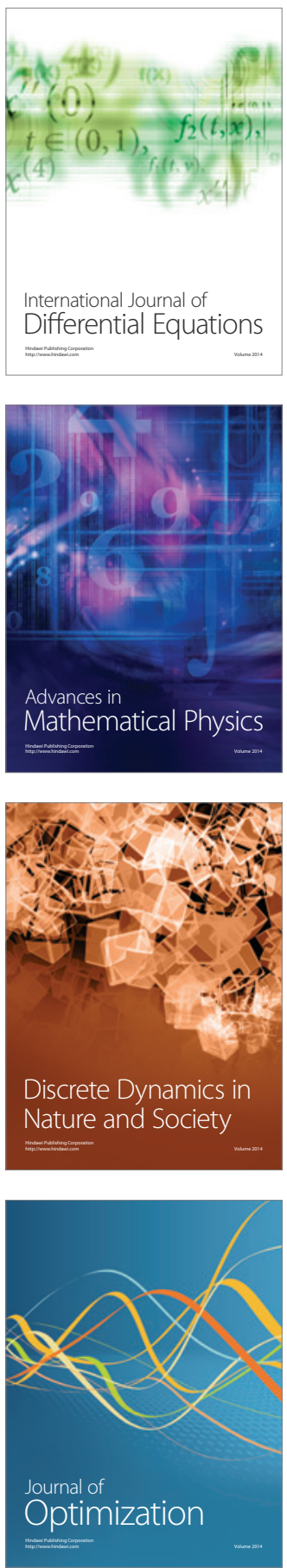